\title{
ASSIMETRIA CAUSAL: UM ESTUDO
}

Túlio Aguiar ${ }^{1}$

RESUMO Neste artigo, examinamos o aspecto assimétrico da relação causal, confrontando-o com o ponto de vista humiano e neo-humiano. Seguindo Hausman e Ehring, favorecemos uma abordagem situacional para a assimetria causal. Nós exploramos a análise do famoso exemplo do mastro (Flagpole), esclarecendo as conexões entre causação e explicação. Nosso diagnóstico geral é que a tradição neo-humiana supõe, equivocadamente, que as relações nômicas, com exceção de pequenos detalhes, exaurem as relações causais.

Palavras-chave: Hume, Hempel, Causação, Explicação.

ABSTRACT This paper examines the asymmetrical aspect of causal relation, confronting it to Humean and Neo-Humean's view. Following Hausman and Ehring, we favor a situational approach to causal asymmetry. We explore the Hausman's analysis of flagpole's example, clearing the connexions between causation and explanation. Our general diagnosis is that the Neo-humean tradition wrongly supposes that nomic relations, with the exception of minor details, exhaust the causal relations.

\section{I}

Hume brindou-nos com uma teoria da causação extraordinariamente bem pensada. Sua teoria é sóbria, em que pretende eliminar o vocabulário causal

1 Universidade Federal de Ouro Preto. Artigo recebido em setembro e aprovado em outubro.

KRITERION, Belo Horizonte, ${ }^{\circ}$ 108, Dez/2003, p.279-289 
com suas noções de poderes, capacidades e necessidades, favorecendo um vocabulário de qualidades sensíveis. Do ponto de vista epistemológico, a teoria é extremamente atraente, pois explica bem o acesso epistêmico à relação causal, uniformizando todos os seus casos por meio da relação de sucessão regular (por exemplo, interações mente-corpo, corpo-corpo, mente-mente). Mais, a teoria presta-se facilmente a uma codificação lógica, com a relação causal sendo assimilada ao condicional material. Ainda, as noções semanticamente suspeitas que constituem o vocabulário causal são eliminadas, mas não sem que antes seja dada uma explicação psicológica de como se originam. Finalmente, estipula-se que a direção da causação coincide com a direção do tempo ${ }^{2}$. A teoria recebe sua expressão mais concisa nas duas definiç̧ões de causa.

$\mathbf{C}_{1}$ : an object precedent and contiguous to another, and where all the objects resembling the former are plac' $d$ in a like relation of priority and contiguity to those objects, that resemble the latter.

$\mathbf{C}_{2}$ : an object precedent and contiguous to another, and so united with it in the imagination, that the idea of the one determines the mind to form the idea of the other, and the impression of the one to form a more lively idea of the other (HUME (1739): 172).

Em sua "Investigação" estas definições se repetem com a notável diferença de que a contigüidade entre os eventos já não é exigida, ecoando talvez a possibilidade de ação a distância apontada pela teoria newtoniana da gravitação ${ }^{3}$. A literatura registra um sem-número de discussões em torno da relação entre as duas definições. Seriam coextensivas? Seriam complementares? Hume teria, realmente, endossado ambas? Quaisquer que sejam as respostas a estas perguntas, aquilo que se constituiu no século XX como a "tradição humiana" investiu pesadamente sobre a versão de $\mathbf{C}_{1}$, em que as relações de contigüidade e prioridade temporal da causa são omitidas, a qual denominaremos $\mathbf{C}_{\mathbf{1}}$ ' Sob a influência do positivismo lógico, $\mathbf{C}_{2}$, de cunho marcadamente psicologista, foi abandonada. Buscou-se um refinamento de $\mathbf{C}_{\mathbf{1}}$ ' que pudesse tornar o enfoque humiano mais preciso e fecundo para tratar do problema das leis $d a$

2 Do ponto de vista da inferência causal, utilizado por Hume para a análise da causação, a situação é simétrica, pois tal inferência pode ir tanto da causa para o efeito, como do efeito para a causa. O prof. Simon Blackburn, em uma intervenção no Colóquio Hume, sugeriu uma interessante maneira de forjar a assimetria da causação dentro da teoria humiana. Segundo ele, devemos observar que as nossas expectativas correm em uma direção bem definida. Assim, a ingestão do pão leva-me à expectativa da saciedade, mas não o contrário. A direção da causalidade seria de natureza puramente mental.

3 Também existe o problema de que no registro mental não é claro como pode haver contigüidade espacial. 
natureza (causação passa para o segundo plano) e de suas ramificações epistemológicas. O lado psicologista da teoria foi substituído por critérios adicionais de cunho lógico, epistemológico e pragmático. À primeira vista, a idéia contida em $\mathbf{C}_{\mathbf{1}}$ ' presta-se de forma exemplar a uma codificação lógica, já que, em uma primeira aproximação, uma relação é nômica se for uma instância de algo do tipo R: (x) $[\mathrm{P}(\mathrm{x}) \mathrm{E} \mathrm{Q}(\mathrm{x})]$. Dois problemas, porém, surgem imediatamente.

Primeiro, $\mathbf{R}$ parece não conter o componente nômico indispensável para capturar as nossas idéias de causa e lei. Afinal de contas, não consideramos que toda regularidade tenha caráter nômico. Por exemplo, a proposição "toda esfera de ouro tem diâmetro menor do que 1000 Km" tem todas as chances de ser uma regularidade universal, sem, contudo, poder qualificar-se como uma lei genuína. Dentro da teoria humiana, talvez este problema encontre uma resposta em $\mathbf{C}_{2}$. Talvez o contexto psicológico determine quais regularidades têm caráter acidental e quais têm caráter nômico, estabelecendo certas redes de semelhança e não outras, sendo este um fato bruto ${ }^{4}$. Uma outra possibilidade, presente embrionariamente em Hume, é apelar para regras gerais que permitam situar convenientemente $\mathbf{R}$, aferindo o seu status nômico. Esta solução não é totalmente distinta da anterior. As regras gerais que formamos a partir de experiência e reflexão poderiam influir no contexto psicológico e explicar como este determina o status de $\mathbf{R}$.

Autores como Goodman e Quine, abstraindo considerações psicológicas, procuram resolver o problema que estamos considerando também em um espírito humiano - atentando para regularidades e para regularidades acerca de regularidades. São nossas práticas indutivas que conferem a $\mathbf{R}$ um certo status. Por exemplo, regularidades utilizadas com êxito para explicar e predizer os fenômenos poderiam alcançar o lugar reservado às leis da natureza, sendo esta determinação a posteriori. Isto é uma tentativa de superar antigas soluções de cunho apriorista e sintaxista, em que as feições lógicas dos predicados contidos em $\mathbf{R}$ (como, por exemplo, seu caráter não-posicional) responderiam por seu caráter nômico (QUINE (1969); GOODMAN (1954)). Qualquer que seja a solução para este problema, focalizaremos, neste artigo, a nossa atenção sobre a componente aparentemente mais inocente da teoria humiana: a prioridade temporal da causa sobre o seu efeito.

4 Boyd, por exemplo, diz: "Hume's account of causation is incomplete without some answer to the question of what respects of resemblances are relevant in applying the definition he offers. It is rather plain that Hume's answer is provided by the "natural" definition of causation: The respects of resemblance that "count" are just those to which the mind naturally attends in forming general beliefs about property correlations"(BOYD (1985): 356). 


\section{II}

Um conceito robusto de causação possui certas feições, por exemplo seu caráter direcional, que são necessárias para resolver importantes problemas epistemológicos. A prioridade temporal é a relação utilizada por Hume para capturar a direção da causação. Mesmo que conseguíssemos qualificar $\mathbf{R}$ de maneira que exista uma ligação nômica entre $\mathbf{P}$ e $\mathbf{Q}$ (ou entre os indivíduos que instanciem estas propriedades), faltaria ainda explicar a dimensão assimétrica da relação causal. Regularidade, conexão necessária, contigüidade são relações simétricas nas quais é indiferente considerar $(a, b)$ ou (b, a). Na relação causal, entretanto, (c, e) é muito diferente de (e, c). Existe uma sub-relação que constitui a relação causal que é verdadeira para (c, e), mas não para (e, c). Em Hume, pelo menos sob a interpretação padrão, é a ordem temporal que responde pela assimetria da relação causal, a causa precede o efeito, mas não vice-versa. Assim, $\mathbf{C}_{\mathbf{1}}$, abstraindo-se o requisito de contiguiidade, pode ser fatorada em duas componentes, a saber, $\mathbf{R}$, que pode receber uma formulação lógica perspícua, mais a estipulação de que a ordem temporal constitui a relação causal. Justiça seja feita, a teoria humiana, tal como expressa em $\mathbf{C}_{\mathbf{1}}$ e $\mathbf{C}_{\mathbf{2}}$, é uma teoria bastante completa. Ela explica a causalidade através de $\mathbf{R}$, do caráter nômico de $\mathbf{R}$ (conferido pelo contexto psicológico) e pela estipulação de que a direção da causação coincide com a direção temporal.

Nas teorias neo-humianas, várias soluções foram apresentadas para explicar o caráter nômico de $\mathbf{R}$. Por exemplo, as feições sintáticas dos predicados contidos em $\mathbf{R}$, características pragmáticas destes predicados como o seu grau de entrincheiramento ${ }^{5}$ (Goodman), a relação sistêmica de $\mathbf{R}$ com o restante de nossas proposições (Lewis, Goodman) ${ }^{6}$. Quanto ao problema da assimetria causal, as teorias neo-humianas têm menos a dizer do que a teoria humiana. Nestas teorias, a ênfase em leis é tão grande que o conceito de causação tornase um pálido conceito subordinado, perdendo as suas características fundamentais. Leis, contrariamente a causação, são simétricas e se se permite que o conceito de causação seja inteiramente absorvido pelo de lei, aquele perde a sua dimensão fundamental. Mesmo em Hume, onde a direção do tempo provê uma solução para o nosso problema, poderíamos nos perguntar se a diferença que a teoria indica entre a causa e o efeito é a diferença correta. Está longe de ser evidente como a diferença temporal pode explicar a diferença entre causa e efeito. Pode ser que, de fato, a causa sempre preceda o seu efeito e que,

5 Simplificando, o grau de entricheiramento de um predicado está relacionado com o número de vezes em que proposições que o contém foram utilizadas com êxito para fazer predições.

6 A respeito destas questões, ver CARROLL (1994). 
portanto, devamos excluir causação retrógrada e simultânea. Isto, porém, pode ser uma contingência e, o que importa para nós, pode não ser a melhor contingência para nos conduzir ao coração do aspecto assimétrico da causação. Devemos lembrar, ainda, que a teoria resulta errônea relacionando efeitos sucessivos de uma causa comum como causa e efeito e que a possibilidade de causação simultânea e retrógrada não deve ser excluídas sem argumento. Assim, tem havido várias tentativas de superar a teoria humiana em direção a uma teoria mais satisfatória. Em seguida, examinaremos algumas sugestões para construir uma teoria da causação alternativa.

Uma boa teoria da causação deve procurar lidar com os aspectos simétricos e assimétricos da causação. Assim procedendo, várias combinações são possíveis quanto as interconexões dos dois aspectos. Pode-se, como na teoria humiana, explicar a componente simétrica (a conexão necessária), reduzindoa a regularidades e, por meio de uma estipulação, fazer a componente assimétrica coincidir com a assimetria temporal. Na tradição neo-humiana, o aspecto assimétrico da causação é absorvido pelo simétrico — o foco recai sobre regularidades nômicas. Uma outra possibilidade presente em algumas teorias contemporâneas é deixar que a componente simétrica seja uma noção primitiva dentro da teoria e buscar alguma elucidação substantiva para a componente assimétrica. Na mesma linha de pensamento, é possível apresentar uma teoria que explique o caráter assimétrico da causação, mostrando que o sucesso desta independe em larga medida da explicação para seu caráter simétrico. Autores como Ehring e Hausman, por exemplo, têm apresentado teorias distintas sobre o aspecto simétrico, mas a mesma idéia fundamental para o aspecto assimétrico da causação. A idéia central, em sua formulação mais neutra, é que dois eventos $a$ e $b$, relacionados de forma a sugerir conexão causal (interpretada como conexão necessária, regularidades ou o que se queira), têm sua função causal determinada por sua relação com outros eventos. Assim, omitindo diversos detalhes, se existe um evento $c$ está conectado a $b$ mas não a $a, a$ causa $b$ ou $a$ e $b$ são efeitos de uma causa comum. Se $c$ está conectado a $a$ mas não a $b$, então $b$ causa $a$ ou $a$ e $b$ são efeitos de uma causa comum. Isto é uma paráfrase e uma síntese de dois princípios apresentados em HAUSMAN (1998): o princípio de conexão ${ }^{7}$ e a condição de independência. A idéia subjacente

7 O princípio de conexão, tal como formulado em HAUSMAN (1998), assevera: "For all events $a$ and $b$, $a$ and $b$ are causally connected if and only if they are distinct and either a causes $b, b$ causes $a$, or $a$ and $b$ are effects of a common cause" (p.59).

A condição de independência diz: "If $a$ causes $b$ or $a$ and $b$ are causally connected only as effects of $a$ common cause, then $b$ has a cause that is distinct from $a$ and not causally connected to $a$ " (p.64).

O seguinte texto esclarece a noção de conexão causal em Hausman: "Reichenbach takes the connection principle to define the notion of a causal connection $(1956$, p. 29). I prefer, in contrast, to take the notion of 
é que fatores causais são em geral causalmente independentes, isto é, eventos que concorrem para causar um terceiro não estão causalmente conectados. Omitindo a possibilidade de que $a$ e $b$ sejam efeitos de uma causa comum e simplificando o cenário para apenas três eventos $a, b$ e $c$, as figuras seguintes mostram como determinar a direção causal com respeito a $a$ - $b$. As linhas que não possuem setas, na fig.1, indicam conexão causal entre os eventos e a linha tracejada indica uma situação alternativa para c. Nas figuras 2 e 3, temos a materialização das duas possibilidades contidas na figura 1 , ou seja, $a$ causa $b$ e $b$ causa $a$, respectivamente.

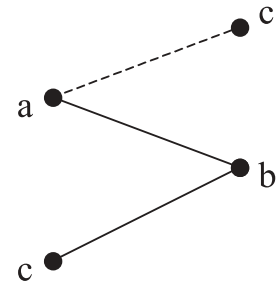

Fig. 1

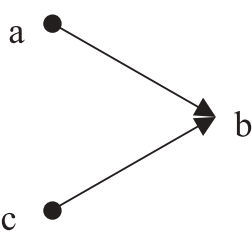

Fig. 2

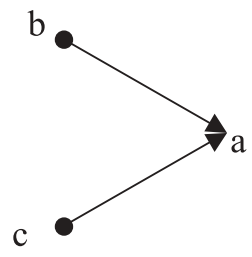

Fig. 3

As considerações acima delineiam, grosso modo, a perspectiva que considera como fundamental, para uma compreensão correta da causação, a situação dos eventos em questão dentro de uma rede de eventos interconectados. É interessante notar que, em contraste com a perspectiva humiana, em que a ênfase está na relação vertical com eventos do mesmo tipo, aqui, o foco está na relação horizontal com eventos de tipos diferentes. Ainda, a abordagem situacional é independente de qual seja o compromisso exato dos diversos autores com relação à possibilidade de reduzir o componente simétrico da causação a regularidades ou com qualquer outra forma de redução. O problema da direção da causação pode ser resolvido mantendo-se neutro em relação à análise precisa de seu aspecto simétrico. Na literatura, encontramos muitas análises diferentes da componente simétrica da causação. Papineau, por exemplo, fala apenas de correlações. Ehring avança uma ambiciosa teoria dos tropos, propriedades particularizadas, cuja persistência explicaria o nexo causal. Já um autor como Hausman prefere trabalhar com uma teoria em que a noção de conexão causal é um termo indefinido. Em todos estes casos o que poderíamos

a causal connection as the undefined intuitive notion of a nomological linkage, that relation among tokens that typically manifests itself as a probabilistic dependency among types" (HAUSMAN (1998): 59) 
chamar de triangulação entra em cena para determinar a direção da causação. Precisamos considerar sempre as relações dos dois eventos em questão com outros eventos relacionados para descobrir a função causal de cada um. Naturalmente, o ponto não é apenas epistemológico. A situação dos eventos dentro da rede constitui uma diferença objetiva, sendo esta a diferença entre causa e efeito.

\section{III}

Uma notável assimetria que pode ser relacionada com a da causação é a assimetria da explicação ${ }^{8}$. Existe uma certa medida de concordância de que podemos explicar efeitos por meio de suas causas, mas não o contrário. $\mathrm{O}$ status da ligação entre causação e explicação, porém, pode variar enormemente. Pode-se estipular simplesmente que explicar consiste em citar uma causa e que isto é tudo. Neste caso, seria interessante apresentar uma teoria razoavelmente precisa e informativa da causação ${ }^{9}$. No outro extremo, podemos considerar causação como uma espécie de explicação; Hempel parece ser um exemplo desta posiçãa ${ }^{10}$. Seria interessante, contudo, avançar mais e ver se é possível descobrir alguma razão substantiva para a assimetria da explicação, tentando liga-la à assimetria da causação. É um ponto, relativamente, incontroverso que a teoria hempeliana sobre explicação reflete a teoria da causação humiana. Examinemos, pois, a adequação do modelo dedutivo-nomológico de Hempel, através de um dos seus supostos contra-exemplos. O exemplo da sombra do mastro ajuda a desenvolver a discussão. Podemos calcular o comprimento da sombra de um mastro (s) se conhecemos a altura deste (h), o ângulo de elevação do sol (a) e o fato de que a luz viaja em linha reta (l). Consideramos também que estes fatos explicam o comprimento da sombra. Acontece que também podemos calcular a altura do mastro se conhecemos o comprimento da sombra e a elevação do sol. Temos neste caso uma explicação? Creio que a maioria das pessoas responderia que não. Não podemos responder a uma pergunta de por que o mastro tem uma certa altura citando a sua sombra e a elevação do sol. O modelo hempeliano de explicação, entretanto,

8 Focalizaremos, neste texto, apenas a questão da explicação de fatos ou eventos singulares. Não estaremos considerando a explicação de leis por meio de leis mais gerais.

9 Nós achamos insuficiente a posição de Van Fraassen, mas aceitamos o seu convite quando diz: "(...) the causal net $=$ whatever structure of relations science describes and leaving to those interested in causation as such the problem of describing that structure in abstract but illuminating ways, if they wish" (VAN FRAASSEN (1980): 124).

10 Putnam, por exemplo, afirma "Hempel himself seems at times to have conceived of his notion of explanation as a replacement for the vernacular concept of causation" (PUTNAM (1999): 139). 
daria as duas derivações como explicativas ${ }^{11}$. Um diagnóstico simples para a questão seria dizer, de forma um tanto frouxa, que o mastro causa a sombra e não o contrário. Isto, porém, deixa a questão na superfície, não mostra qual é a diferença entre a causa e o efeito que é relevante para as nossas práticas explanatórias.

Vejamos se a teoria da causação delineada na seção anterior pode ajudar. Dentre as variáveis que estão em jogo apenas a altura do mastro (h) e o ângulo de elevação do sol (a) são causalmente independentes. Como já dissemos, a noção de conexão causal está sendo tomada sem análise. Para alguém com escrúpulos quanto a esta noção, deve-se notar que a noção de conexão causal pode ser aproximada pela de dependência probabilística. Assim, o ângulo de elevação do sol e a altura do mastro são probabilisticamente (e causalmente) independentes. Isto não ocorre para as outras combinações das variáveis - o ângulo de elevação do sol e o comprimento da sombra, de um lado, e, de outro, o comprimento da sombra e a altura do mastro são causalmente (probabilisticamente) dependentes. Qual o impacto destas observações para uma teoria da explicação? As variáveis h e a são, por assim dizer, separáveis e podem ser combinadas a vontade para gerar s. Já se considerarmos s e a, não podemos combina-las com a mesma liberdade para gerar $\mathbf{h}$. Não podemos, por exemplo, variar uma mantendo a outra fixa e isto de alguma forma empobrece nossa compreensão do fato a ser explicado. Na literatura sobre o assunto, freqüentemente, requer-se que uma explicação desvele o mecanismo pelo qual o explanandum é produzido. Também é comum pensar que uma boa explicação deve unificar o nosso conhecimento. Quanto ao primeiro aspecto, Hausman argumenta que a independência causal das variáveis envolvidas está implícita na noção de um mecanismo (HAUSMAN (1998): 173). É como se precisássemos identificar separadamente a capacidade causal de cada componente para compreendermos como se combinam para produzir um resultado. Quando partimos do efeito, partimos de algo que já contém, por assim dizer, uma mistura e não podemos dar uma explicação mecânica. Quanto à unificação, teremos um maior poder unificador à medida que conseguirmos explicar uma grande variedade de fatos com o menor número de elementos simples. Por esta razão não é uma boa explicação utilizar a sombra do mastro e o ângulo de elevação do sol para a explicar a altura do mastro, pois neste caso há sobreposição de elementos, sendo este fato refletido na conexão causal entre o comprimento da sombra e o ângulo de elevação do sol. Ecoando o tema de um texto de Gasking,

11 Como pode ser comprovado em HEMPEL (1965): 352-353 e HEMPEL (1962): 94-95. 
intitulado "Causation and Recipes", Hausman apresenta um sugestivo comentário sobre o tema:

When it (the independence condition) is not satisfied, there can be no recipes. It is not possible separately to add a little more salt. A certain kind of explanation for the flavor of the soup cannot be given. Without independence, there is no basis for defining asymmetrical relations among nomological connections and no asymmetrical relations to be defined. Causes are simply independent factors (HAUSMAN(1998): 175).

Tudo isto mostra que a existência de um elo nomológico entre dois itens definitivamente não é o bastante para se compreender a causação. É preciso se fazer justiça a seu caráter assimétrico e a teoria apresentada acima parece estar no caminho certo.

Um outro tipo de requisito para uma explicação, devido a Hempel, estabelece uma ligação desta com a noção de preditibilidade. Sinteticamente, os fatos contidos no explanans permitiriam a predição do explanandum. No exemplo da sombra do mastro, o valor de quaisquer duas variáveis leva a predição da terceira. $\mathrm{O}$ mesmo não acontece, porém, se o foco recai sobre a conseqüência de mudanças sobre as variáveis. Se variarmos, por exemplo, o tamanho do mastro, podemos, supondo o restante igual, predizer o novo valor para a sombra. Por outro lado, se a sombra varia, não obteremos o valor correto para a altura do mastro, considerando o resto igual. Se a sombra varia, apenas um valor para a elevação do sol permitirá a derivação correta da altura do mastro. Além disso, no primeiro caso, podemos responder a uma ampla gama de perguntas representadas por uma classe de contrafactuais do tipo "o que ocorreria se $\boldsymbol{h}$ tivesse o valor $\boldsymbol{h}$ ' e $\boldsymbol{a}$ tivesse o valor $\boldsymbol{a}$ " "? No segundo caso, tais perguntas não fazem sentido. Assim, quando a situação deixa de ser estática e as variáveis não são independentes há um declínio do poder preditivo do explanans, ocorrendo, também, uma diminuição da capacidade de combinação de suas variáveis com conseqüente diminuição da gama de perguntas que podem ser feitas e respondidas. Uma explicação não apenas responde a uma pergunta, ela mobiliza os elementos apropriados para responder a um amplo espectro de perguntas possíveis. Por esta razão, nem toda dedução que tenha o explanandum como conclusão se qualifica como uma boa explicação.

\section{IV}

Existem exemplos extremos em que a teoria esboçada acima encontra dificuldades. Por exemplo, num universo com apenas duas partículas que interagem gravitacionalmente, parece difícil construir algum tipo de triangulação 
para se determinar a direção causal. Neste caso, pode-se introduzir eventos negativos como faz Ehring e assim efetuar a triangulação. Uma outra possibilidade consiste em negar a existência de relação causal neste tipo de situação, embora podendo admitir a existência de relações nômicas simétricas. Esta possibilidade parece ser o que Hausman tem em mente quando diz que é impróprio falar de relações causais em sistemas fechados (HAUSMAN (1998): 145-146). Relações causais existem em sistemas abertos, caracterizados pela possibilidade de intervenções de um certo tipo. Intervenções para serem efetivas precisam incidir sobre uma estrutura em que existem fatores causais independentes. Por exemplo, se no processo de alimentarmos certos ratos com sacarina a nossa escolha das cobaias estiver correlacionada com algo ligado a sua tendência de desenvolver câncer, não estaremos realmente intervindo. Não podemos dizer que o desenvolvimento posterior do câncer é efeito da dieta de sacarina. É por isso que as amostras devem ser obtidas aleatoriamente, para que possamos assegurar independência entre as variáveis (no caso, a tendência para o câncer e a dieta de sacarina) e determinar a ordem causal.

A discussão acima indica a importância de se dar um lugar próprio para as relações causais, diferenciando-as de relações meramente nomológicas. Pelo menos parte da diferença consiste no fato de que a causalidade é uma relação direcional, contrariamente às relações nomológicas. Podemos ver também como se pode avançar em relação à teoria humiana, ou pelo menos neo-humiana, colocando sob suspeita o fato de que a relação de precedência temporal marque a diferença fundamental entre causa e efeito, ainda que de fato tal precedência sempre exista. Considerando as relações entre causação e explicação, fica evidenciado como é possível diagnosticar parte do problema com o modelo dedutivo-nomológico de Hempel cuja inserção na tradição humiana é consensual. É claro que muito falta para uma teoria completa da causação e da explicação. Um dos passos para completá-la poderia ser a elucidação da noção de ligação nomológica. Mais importante talvez é o desenvolvimento de uma teoria que explique o componente singularista da causação, isto é, aquele componente local que responde pela ligação de dois eventos ou propriedades específicas, permitindo o emparelhamento da causa e do efeito ${ }^{12}$. Em uma teoria de tipo humiano, tal componente singularista é dada pela contigüidade no espaço e no tempo ${ }^{13}$. Teorias como as de Ehring e Salmon apelam a formas de

12 Por exemplo, a regularidade "o pão alimenta" não permite emparelhar corretamente um determinado tokencausa com o seu correspondente token-efeito. Nós teríamos que excluir a possibilidade que este pão que estou comendo cause a saciedade em outra pessoa, daí a necessidade da contigüidade no espaço e no tempo.

13 Isto mostra que a teoria humiana, realmente, precisa do requerimento de contigüidade. 
persistência e transferência entre os itens que entram na relação causal. Isto, obviamente, nos remete inevitavelmente a ontologia da causação - que espécies de coisas, exatamente, estariam nesta relação? Devemos nos lembrar do velho exemplo que vem de um contexto um pouco diferente. "Todas as coisas não-negras são não-corvos" pode ser assumida como um exemplo de regularidade nômica. É uma questão árdua saber o que falta exatamente para convertê-la em uma regularidade causal.

\section{Bibliografia}

BOYD, R. (1985). “Observations, Explanatory Power, and Simplicity: Toward a NonHumean Account" in BOYD ET ALLI (eds.). The Philosophy of Science. Cambridge (Massachusetts), MIT Press, 1997.

CARROLL, J. (1994). Laws of Nature. Cambridge, Cambridge University Press.

DOWE, P. (1996). "Backwards Causation and the Direction of Causal Processes". Mind, 105, pp. 227-248.

EHRING, D. (1997). Causation \& Persistence: a Theory of Causation. Oxford, Oxford University Press.

GOODMAN, N. (1954). Fact, Fiction, and Forecast. Cambridge, Harvard University Press.

HAUSMAN, D. (1998). Causal Asymmetries. Cambridge, Cambridge University Press. HUME, D. (1978). A Treatise of Human Nature. Oxford, Oxford University Press.

HUME, D. (1999). An Enquiry Concerning Human Understanding. Oxford, Oxford University Press.

PUTNAM, H. (1999). The Threefold Cord. New York, Columbia University Press, 2001.

QUINE, W.O. (1969). "Natural Kinds” in KORNBLITH, H. (ed.). Naturalizing Epistemology. Cambridge, The MIT Press, 1994.

REICHENBACH, H. (1957). The Direction of Time. Mineola, Dover Publications.

SALMON, W. C. (1998). Causality and Explanation. Oxford, Oxford University Press. 